\title{
Low neutrophil alkaline phosphatase score is a new aspect of calreticulin-mutated myeloproliferative neoplasms
}

\author{
Toshinori Kondo ${ }^{1 *}$, Taizo Tasaka ${ }^{1,3}$, Nanako Tomioka², Fuminori Sano ${ }^{1}$, Hirotoshi Tokunaga', \\ Shin-ichiro Suemori ${ }^{2}$, Takayuki Tsujioka ${ }^{2}$, Yoshiko Matsuhashi ${ }^{1}$, Hidekazu Nakanishi ${ }^{1}$, Hideho Wada', \\ Kaoru Tohyama ${ }^{2}$ and Takashi Sugihara ${ }^{1}$
}

\begin{abstract}
Calreticulin (CALR) and JAK2-V617F gene mutations, which are major genetic mutations in patients with primary myelofibrosis (PMF) and essential thrombocythemia (ET), exert different effects on the clinical features and outcomes of these diseases. We analyzed 88 and 9 patients with ET and PMF, respectively, and determined the differences in the clinical characteristics of ET patients with JAK2-V617F compared with CALR mutations. The frequency of the JAK2V617F and CALR mutations were 64 and $22 \%$, respectively. Patients with CALR mutations were younger, had a lower white blood cell count, and had a lower rate of thrombotic events than patients with the JAK2 mutation. The neutrophil alkaline phosphatase (NAP) score of 16 patients with CALR mutations was significantly lower than the normal controls, which was mainly due to the high proportion of NAP-negative neutrophils. This is the first report to show an association between CALR mutations in patients with myeloproliferative neoplasms (MPN) and the NAP score. Although the mechanism is unclear, the NAP score could be a useful and reliable biochemical marker to discriminate the mutational status of MPN patients. Further investigation is warranted to determine whether these characteristics contribute to the pathogenesis of MPN and the NAP score.
\end{abstract}

Keywords: Myeloproliferative neoplasms, Calreticulin, Alkaline phosphatase score, Essential thrombocythemia, Janus kinase 2

\section{Background}

The pathogenesis of myeloproliferative neoplasms (MPN) is characterized by constitutive activation of "disease-specific" protein tyrosine kinases. The Janus kinase 2 (JAK2)V617F mutation and somatic mutations of JAK2 exon 12 constitutively activate JAK2 protein tyrosine kinase and these mutations are detected in almost all patients with polycythemia vera (PV). In addition, the JAK2V617F mutation is detected in approximately $50-60 \%$ of patients with essential thrombocythemia (ET) and primary myelofibrosis (PMF) (Baxter et al. 2005; James et al. 2005; Kralovics et al. 2005; Levine et al. 2005; Campbell

\footnotetext{
*Correspondence: kondot@med.kawasaki-m.ac.jp

1 Department of Hematology, Kawasaki Medical School, 577 Matsushima, Kurashiki City, Okayama Prefecture 701-0192, Japan

Full list of author information is available at the end of the article
}

and Green 2006), and activating mutations of the myeloproliferative leukemia virus oncogene $(M P L)$ are present in $5-10 \%$ of patients with ET or PMF without the JAK2V617F mutation (Pikman et al. 2006; Rumi et al. 2013). Furthermore, novel mutations in the gene encoding calreticulin $(C A L R)$ were discovered in patients with $\mathrm{ET}$ and PMF with wild-type $J A K 2$ or MPL (Klampfl et al. 2013; Nangalia et al. 2013). CALR mutations are detected only in patients with ET or PMF (Klampfl et al. 2013; Nangalia et al. 2013). The frequency of CALR mutations in patients with ET and PMF are 25 and $35 \%$, respectively. The clinical features of ET patients with CALR mutations include a higher platelet count, lower hemoglobin level and white blood cell count, fewer thrombotic events, and less leukemic transformation compared to ET patients with JAK2V617F mutation (Rumi et al. 2014). These results suggest 
that the clinical features and outcomes of MPN patients are defined by the gene alterations that they acquired.

The neutrophil alkaline phosphatase (NAP) score is a useful and reliable biochemical marker that supports the diagnosis of MPN (Bendix-Hansen and Bergmann 1985). The chronic phase of chronic myelogenous leukemia (CP-CML) is characterized by a low NAP score that is elevated when the disease enters the accelerated phase (Kaplow 1971). Some MPN patients with JAK2V617F mutation have higher NAP scores. Although the NAP score often reflects the pathophysiological status of patients with MPNs, to our knowledge, the details of NAP scores of patients with MPN with CALR mutations have never been studied. Therefore, the goal of the present study was to identify the clinical features of MPN patients with $J A K 2-\mathrm{V} 617 \mathrm{~F}$ and $C A L R$ mutations by focusing on their NAP scores.

\section{Methods}

\section{Patients}

We recruited 88 patients with ET and 9 with PMF. Patients were diagnosed according to the criteria of the 2008 WHO classification. Clinical laboratory findings acquired at the first visit were utilized. The ethics committees of Kawasaki Medical School and Kawasaki Medical School Hospital (Kurashiki, IRB No. 1747 and 1769) approved this study, and all patients provided written informed consent.

\section{NAP score analysis}

The NAP score was determined using a peripheral blood smear stained using our laboratory's protocol. Briefly, slides with dried peripheral blood smears were fixed for $5 \mathrm{~s}$ in ice-cold methanol containing $10 \%$ formalin and $0.001 \%$ glacial acetic acid. After drying, the slides were treated with a staining solution $(0.13 \mathrm{mmol} / \mathrm{l}$ naphthol AS-MX phosphate, $0.25 \mathrm{~mol} / \mathrm{l}$ dimethylformamide, $0.076 \mathrm{~mol} / \mathrm{l}$ propanediol buffer, and $0.04 \mathrm{mmol} / \mathrm{l}$ Fast Blue RR Salt) at $37{ }^{\circ} \mathrm{C}$ for $2 \mathrm{~h}$. After washing with water, the slides were stained using $1 \%$ safranin for $2 \mathrm{~min}$. Neutrophils $(n=100)$ were assigned scores ranging from 0 to 5 according to the number and distribution of NAP-positive granules. The median NAP score of a healthy control in our laboratory is 250 .

\section{DNA sequence analysis}

Genomic DNA was isolated from polymorphonuclear leukocytes of patients' peripheral blood. We tested for $J A K 2, M P L$, and $C A L R$ mutations that were previously reported in patients with ET or PMF (Kondo et al. 2008). The primers, which were designed using Primer3 (version 0.4.0) software (accessible at http://primer3.sourceforge.net/), used to detect the mutations were as follows (forward, reverse): JAK2V617F, 5'-AGTCATGCTGAAAGTAGGA-3' and 5'-ATTGCTTTCCTTTTTCACAAGAT-3'; CALR exon 9, 5'-CTGGTCCTGGTCCTGATGTC-3' ${ }^{\prime}$ and 5'-CAGAGACATTATTTGGCGCG-3'; and $M P L$ exon 10, 5'-AGAGTAGGGGCTGGCTGGAT-3' and 5'-CAGGTCCCACCTCCTAAACC-3'. DNA was amplified using polymerase chain reaction (PCR) Master Mix (Promega $\mathrm{KK}$, Madison, WI, USA) as follows: 1 cycle at $95{ }^{\circ} \mathrm{C}$ for $2 \mathrm{~min}, 35$ cycles of denaturation at $95^{\circ} \mathrm{C}$ for $30 \mathrm{~s}$, annealing at $52{ }^{\circ} \mathrm{C}\left(M P L, 60^{\circ} \mathrm{C}\right)$ for $30 \mathrm{~s}$, and extension at $72{ }^{\circ} \mathrm{C}$ for $30 \mathrm{~s}$. PCR products were analyzed using $3 \%$ agarose gel electrophoresis in the presence of ethidium bromide, and the amplicons were purified using Illustra ExoProS$\operatorname{tar}$ (GE Healthcare Life Sciences, Buckinghamshire, UK). Direct sequencing was performed using the Big Dye Terminator ver3.1 Cycle Sequencing Kit (Applied Biosystems, Foster City, CA, USA) and an ABI Prism 3130 Genetic Analyzer. Sequence data were analyzed using Mutation Surveyor ver3.10 (Softgenetics, State College, PA, USA).

Subjects' characteristics and clinical parameters were compared using the $\chi^{2}$ test, Mann-Whitney $U$ test, and Kruskal-Wallis tests. The strength of the association between two variables was determined using the Spearman's rank correlation. Probability values $<0.05$ were considered significant. Statistical analyses were performed using SPSS ver.15.0.1 J software (IBM, Tokyo, Japan).

\section{Results}

We searched for $J A K 2, C A L R$, and $M P L$ mutations in 88 patients with ET and 9 patients with PMF. Table 1 shows the clinical and laboratory features at diagnosis of the patients stratified according to MPN subtype and mutational status. In the 88 patients with ET, the frequency of the $J A K 2-\mathrm{V} 617 \mathrm{~F}$ and $C A L R$ exon-9 mutations were 65 and $21 \%$, respectively, and in the patients with PMF, they were 56 and $22 \%$, respectively. MPL mutations were not detected in any patient. Triple negativity for $J A K 2, C A L R$, and $M P L$ mutations was found in 14 and $22 \%$ of the patients with ET and PMF, respectively. CALR mutations were detected in 21 patients, and the details of these mutations are shown in Table 2. All mutations were heterogeneous insertions or deletions in exon 9, with 10 distinct variants as follows: six deletions, two insertions, and two complex insertions and deletions. All CALR mutations were predicted to generate a commonly known C-terminal peptide sequence (Klampfl et al. 2013; Nangalia et al. 2013). There were two common variants: L367 fs*46 (33 \%) and K385 fs*47 (24\%).

We investigated the clinical features of ET patients with $J A K 2$ and CALR mutations. The median age of ET patients with $C A L R$ mutations was less than the ET patients with $J A K 2$ mutations, although this difference was not 
Table 1 Presenting features of the 97 patients with ET or PMF, stratified according to their mutational status

\begin{tabular}{|c|c|c|c|c|}
\hline & $J A K 2$ mutated (A) & CALR mutated (B) & Triple negative & $p(A \text { vs } B)^{*}$ \\
\hline \multicolumn{5}{|l|}{$\mathrm{ET}(\mathrm{N}=88)$} \\
\hline No. of patients, n (\%) & $57(65)$ & $19(21)$ & $12(14)$ & \\
\hline Age, median (range), year & $66(33-86)$ & $58(30-85)$ & $66.5(29-82)$ & 0.056 \\
\hline Age $\geqq 60$ year, $\mathrm{n}(\%)$ & $42(74)$ & $8(42)$ & $8(67)$ & 0.012 \\
\hline Females, n (\%) & $33(58)$ & $9(47)$ & $5(42)$ & 0.439 \\
\hline Leukocytes, median (range), $\times 10^{9} / \mathrm{L}$ & $12.1(6.0-42.3)$ & $9.2(5.3-16.3)$ & $8.4(4.6-13.9)$ & $<.001$ \\
\hline Hemoglobin, median (range), g/dL & $14.3(10.2-18.6)$ & $13.5(11.4-17.1)$ & $13.7(11.3-16.2)$ & 0.593 \\
\hline Platelets, median (range), $\times 10^{9} / \mathrm{L}$ & 867 (489-2285) & $930(631-2336)$ & $717(501-922)$ & 0.359 \\
\hline NAP score median (range) & $312(202-417)$ & $215(106-286)$ & $254.5(116-404)$ & $<.001$ \\
\hline High LD, n (\%) & $38(67)$ & $11(58)$ & $3(25)$ & 0.489 \\
\hline Palpable splenomegaly, n (\%) & $19(33)$ & $3(16)$ & $1(8)$ & 0.144 \\
\hline Thrombosis history, n (\%) & $22(39)$ & $2(11)$ & $3(25)$ & 0.023 \\
\hline Needed to cytoreductive therapy, n (\%) & $39(68)$ & $12(63)$ & $5(42)$ & 0.672 \\
\hline \multicolumn{5}{|l|}{$\operatorname{PMF}(\mathrm{N}=9)$} \\
\hline No. of patients, n (\%) & $5(56)$ & $2(22)$ & $2(22)$ & \\
\hline Age, median (range), year & $65(51-85)$ & $63.5(58-69)$ & $67.5(57-78)$ & NA \\
\hline Age $\geqq 60$ year, $n(\%)$ & $4(80)$ & $1(50)$ & $1(50)$ & NA \\
\hline Females, n (\%) & $3(60)$ & $1(50)$ & $1(50)$ & NA \\
\hline Leukocytes, median (range), $\times 10^{9} / \mathrm{L}$ & $14.4(8.6-37.1)$ & $5.8(5.6-6.1)$ & $5.1(5.0-5.3)$ & NA \\
\hline Hemoglobin, median (range), g/dL & $13.2(9.8-16.8)$ & $12.2(11.7-12.6)$ & $11.1(8.9-13.3)$ & NA \\
\hline Platelets, median (range), $\times 10^{9} / \mathrm{L}$ & $384(194-654)$ & $306(260-352)$ & $383(122-644)$ & NA \\
\hline NAP score median (range) & $341(301-378)$ & $157(150-164)$ & $253.5(210-297)$ & NA \\
\hline High LD, n (\%) & $5(100)$ & $2(100)$ & $1(50)$ & NA \\
\hline Palpable splenomegaly, n (\%) & $5(100)$ & $2(100)$ & $0(0)$ & NA \\
\hline Thrombosis history, n (\%) & $1(20)$ & $0(0)$ & $1(50)$ & NA \\
\hline Needed to cytoreductive therapy, n (\%) & $1(20)$ & $0(0)$ & $1(50)$ & NA \\
\hline
\end{tabular}

NA Not available

${ }^{*}$ Calculated using the $x^{2}$ test except for age, CBC and NAP score, which was calculated using the Mann-Whitney $U$ test

significant $(p=0.056)$. The proportion of patients aged $>60$ years was significantly lower in the CALR mutation group $(p=0.012)$. The ET patients with CALR mutations had a significantly lower white blood cell count $(p<0.001)$, NAP score $(p<0.001)$, and prevalence of thrombotic events than the patients with $J A K 2$ mutations ( $p=0.023$; Table 1).

The NAP scores of 63 patients with MPN were evaluable, including 47 patients with $J A K 2$ mutations and 16 patients with CALR mutations. The median NAP score of the patients with $C A L R$ mutations was significantly lower than normal controls (Fig. 1). In contrast to the normal to high range of NAP scores of the patients with JAK2 mutations, the scores of the patients with CALR mutations were normal to low. The NAP rate of the MPN patients with $C A L R$ mutations was lower $(n=13$; median NAP rate $55 \%$; range $38-91 \%$ ), mainly due to the high proportion of NAP-negative neutrophils (score $=0$; Fig. 2). This pattern of NAP scores was similar to that of patients with CP-CML with low NAP scores $(n=9$; median NAP rate $51 \%$; range $20-67 \%$; Fig. 2). There was no significant correlation between the NAP score and any clinical parameter (white blood cell count, hemoglobin value, platelet count, or disease duration) in the MPN patients with CALR mutations (data not shown). The NAP rate of the MPN patients with JAK2 mutations was high compared with patients with $C A L R$ mutations $(n=10$; median $87.5 \%$; range $74-100 \%$ ) and may be explained by the increase in strongly NAP-positive neutrophils (score 4 or 5 ). In patients with $J A K 2$ mutations, the NAP score was significantly correlated with the burden of the V617F mutant allele $(n=47, p<0.001, r=0.748)$.

\section{Discussion}

In 2013, two groups reported the discovery of CALR mutations in patients with ET or PMF (Klampfl et al. 2013; Nangalia et al. 2013). In the present study, the frequencies of CALR mutations in patients with ET and PMF were 21 and $22 \%$, respectively. The frequencies of $C A L R$ L367 fs"46 and K385 fs*47 mutations, which are the most 
Table 2 Mutational status of CALR exon 9 in the 21 patients with ET or PMF

\begin{tabular}{|c|c|c|c|}
\hline Diagnosis & cDNA change & Protein change & C-terminal novel amino acid sequence \\
\hline ET & c.1099_1150del & p.L367 fs*46 & TRRMMRTKMRMRRMRRTRRKMRRKMSPARPRTSCREACLQGWTEA \\
\hline ET & c.1099_1150del & p.L367 fs*46 & TRRMMRTKMRMRRMRRTRRKMRRKMSPARPRTSCREACLQGWTEA \\
\hline PMF & c.1099_1150del & p.L367 fs*46 & TRRMMRTKMRMRRMRRTRRKMRRKMSPARPRTSCREACLQGWTEA \\
\hline ET & c.1092_1143del & p.L367 fs*46 & TRRMMRTKMRMRRMRRTRRKMRRKMSPARPRTSCREACLQGWTEA \\
\hline ET & c.1099_1150del & p.L367 fs*46 & TRRMMRTKMRMRRMRRTRRKMRRKMSPARPRTSCREACLQGWTEA \\
\hline ET & c.1099_1150del & p.L367 fs*46 & TRRMMRTKMRMRRMRRTRRKMRRKMSPARPRTSCREACLQGWTEA \\
\hline ET & c.1099_1150del & p.L367 fs*46 & TRRMMRTKMRMRRMRRTRRKMRRKMSPARPRTSCREACLQGWTEA \\
\hline ET & c.1154_1155ins TTGTC & p.K385 fs*47 & NCRRMMRTKMRMRRMRRTRRKMRRKMSPARPRTSCREACLQGWTEA \\
\hline PMF & c.1154_1155ins TTGTC & p.K385 fs*47 & NCRRMMRTKMRMRRMRRTRRKMRRKMSPARPRTSCREACLQGWTEA \\
\hline ET & c.1154_1155ins TTGTC & p.K385 fs*47 & NCRRMMRTKMRMRRMRRTRRKMRRKMSPARPRTSCREACLQGWTEA \\
\hline ET & c.1154_1155ins TTGTC & p.K385 fs*47 & NCRRMMRTKMRMRRMRRTRRKMRRKMSPARPRTSCREACLQGWTEA \\
\hline ET & c.1154_1155ins TTGTC & p.K385 fs*47 & NCRRMMRTKMRMRRMRRTRRKMRRKMSPARPRTSCREACLQGWTEA \\
\hline ET & c.1102_1153del & p.K368 fs*45 & RRMMRTKMRMRRMRRTRRKMRRKMSPARPRTSCREACLQGWTEA \\
\hline ET & c.1102_1153del & p.K368 fs*45 & RRMMRTKMRMRRMRRTRRKMRRKMSPARPRTSCREACLQGWTEA \\
\hline ET & c.1110_1140del & p.E371 fs*49 & RQRTRRMMRTKMRMRRMRRTRRKMRRKMSPARPRTSCREACLQGWTEA \\
\hline ET & c.1109_1160del & p.E370 fs*42 & VMRTKMRMRRMRRTRRKMRRKMSPARPRTSCREACLQGWTEA \\
\hline ET & c.1150_1154delins TGTC & p.D384 fs*46 & CRRMMRTKMRMRRMRRTRRKMRRKMSPARPRTSCREACLQGWTEA \\
\hline ET & c.1125_1126 ins TTCTTAGTGCT & p.R376 fs*58 & FLVLAKRRRRQRTRRMMRTKMRMRRMRRTRRKMRRKMSPARPRTSCREACLQGWTEA \\
\hline ET & 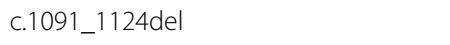 & p.E364 fs*54 & DAKRRRRQRTRRMMRTKMRMRRMRRTRRKMRRKMSPARPRTSCREACLQGWTEA \\
\hline ET & c.1080_1144delins GGAAGAAGACAAG & p.Q361 fs*51 & $\begin{array}{l}\text { KAAEKQMKDKEEDKQRTRRMMRTKMRMRRMRRTRRKMRRKMSPARPRTSCREA- } \\
\text { CLQGWTEA }\end{array}$ \\
\hline ET & c.1122_1134del & p.K375 fs*50 & $\begin{array}{l}\text { KAAEKQMKDKQDEEQRLKEEEEDKRRRQRTRRMMRTKMRMRRMRRTRRKMRRKM- } \\
\text { SPARPRTSCREACLQGWTEA }\end{array}$ \\
\hline
\end{tabular}

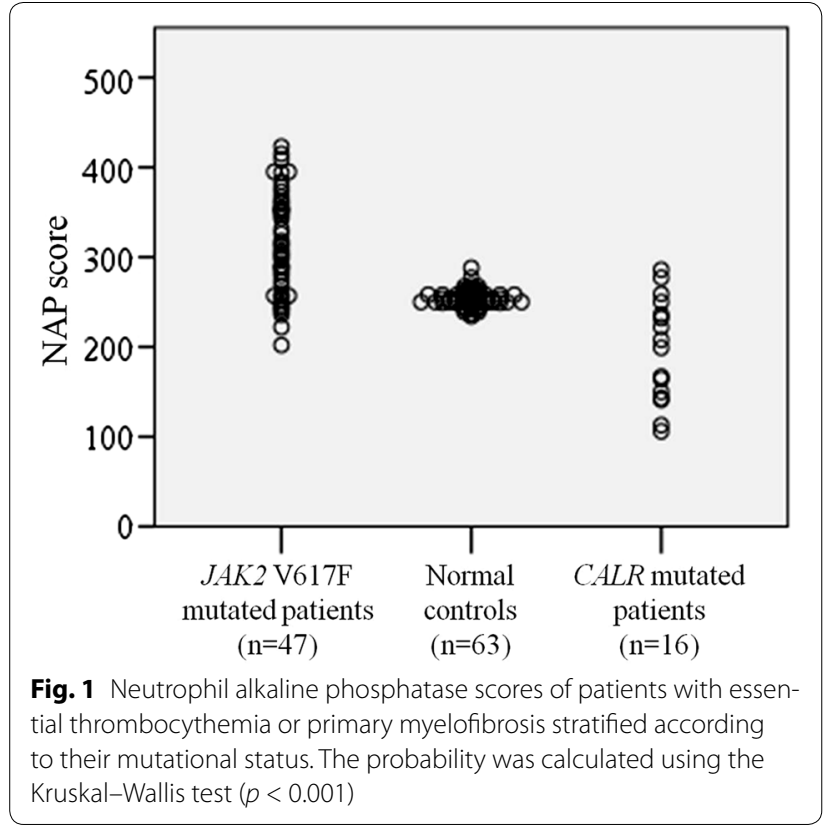

common mutations in Western countries (Klampfl et al. 2013; Nangalia et al. 2013), were 33 and $24 \%$, respectively, in our series. Patients with CALR mutations are younger, have a lower hemoglobin level and white blood cell count, and have a higher platelet count and serum erythropoietin than patients with JAK2-V617F mutation (Rumi et al. 2014). CALR mutations can also affect the clinical outcomes. ET patients with CALR mutations do not transform to PV (Rumi et al. 2014). In our series, the clinical features of the patients with CALR mutations included younger age, decreased white blood cell count, and a lower rate of thrombosis compared with patients with JAK2 mutations, although there were no significant differences in hemoglobin levels or platelet count. Further studies are required to better understand the phenotypes of patients with CALR mutations. Altogether, the available data suggest that the differences in mutational status define the clinical subtypes of the disease.

We found that the NAP score of patients with $J A K 2$ mutations generally ranged from normal to high levels and was correlated with their JAK2-V617F mutant-allele burden. In contrast, the median NAP score of the 16 patients with $C A L R$ mutations was significantly lower than the normal controls. To our knowledge, this is the first study to report low NAP scores in patients with CALR mutations, which were mainly due to the high proportion of NAP-negative neutrophils and represents a new biological aspect of MPN patients with CALR mutations. Therefore, the NAP score 


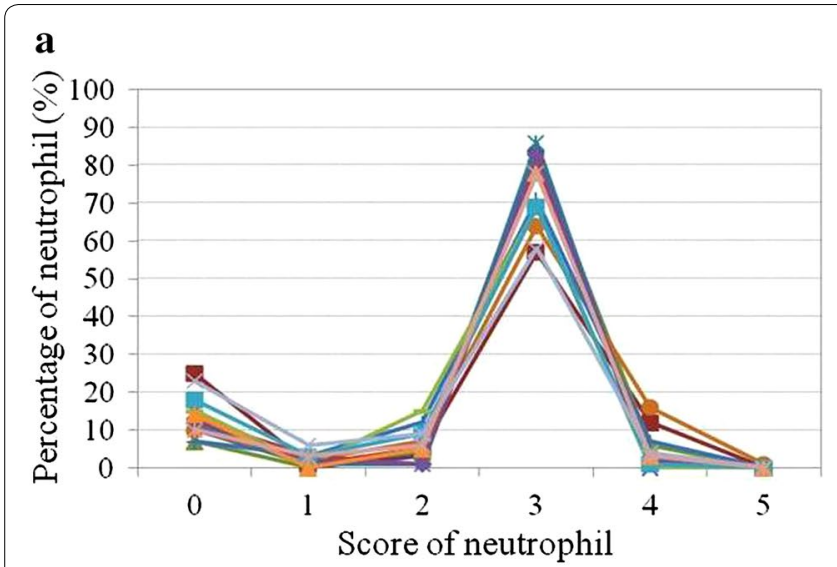

c

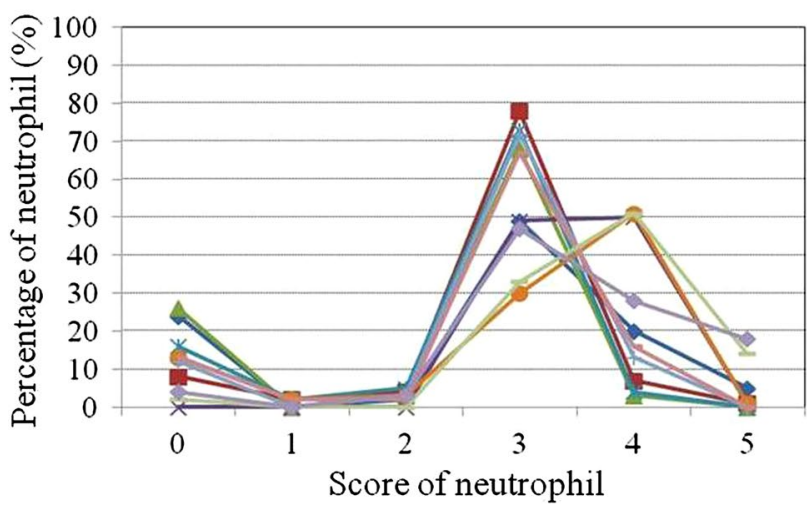

\section{b}

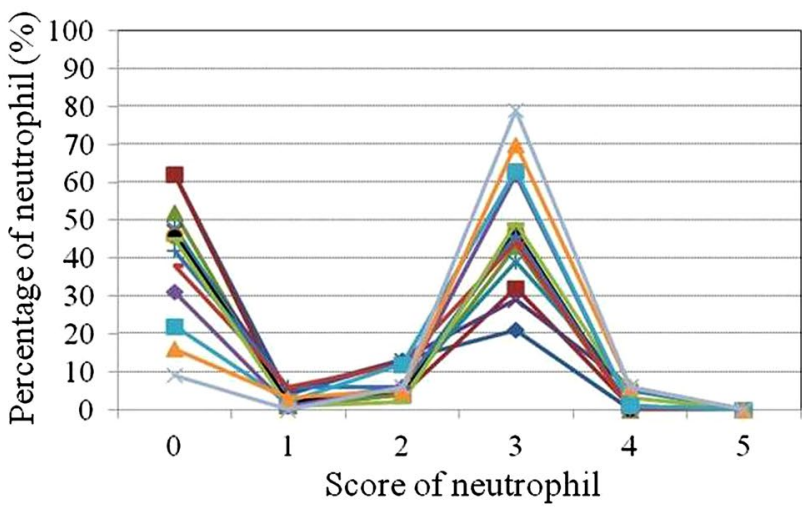

d

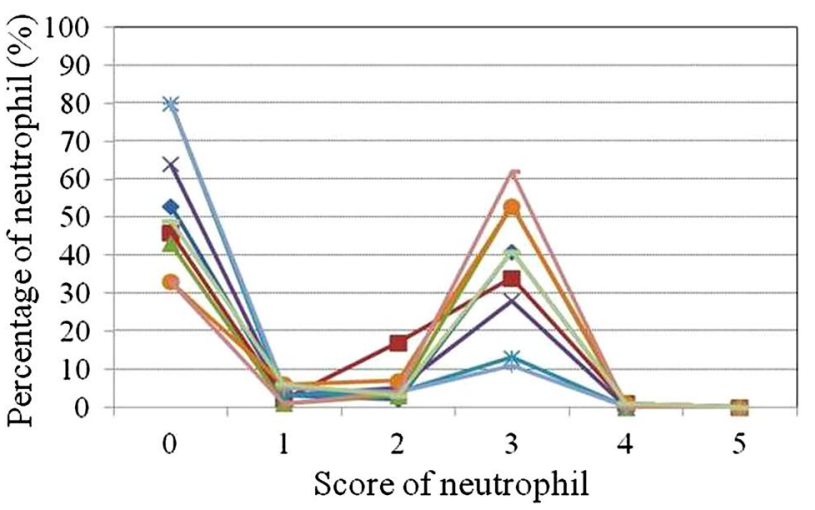

Fig. 2 Analysis of the neutrophil alkaline phosphatase rate according to the mutational status of patients with MPN. a Normal controls ( $n=14$ ). b Patients with MPN with CALR mutations $(n=13)$. c Patients with MPN with JAK2-V617F mutation $(n=10)$. d Patients in the CP-CML $(n=9)$. One hundred neutrophils were counted in each sample

could be a useful and reliable biochemical marker to discriminate the mutational status in patients with ET or PMF.

In patients with CP-CML, a low NAP score is the result of low serum levels of granulocyte colony-stimulating factor (Yuo et al. 1987; Watari et al. 1989; Saitoh and Shibata 1993; Tsushita et al. 1993). In contrast, in the present study, there was no significant correlation between these variables (data not shown). Several reports describe the relationship between NAP activation and MPN-related mutations. For example, transfection of the NB4 cell line with a construct that expresses $J A K 2-\mathrm{V} 617 \mathrm{~F}$ induces cell proliferation through the phosphorylation of the signal transducer and activator of transcription (STAT) 5, leading to phosphorylation of STAT3, which activates NAP expression (Oku et al. 2010). Furthermore, patients with MPN, regardless of their diagnosis or JAK2 mutational status, are characterized by upregulation of JAK-STAT signaling molecules, and the CALR mutation activates the JAK2-STAT5 signaling pathway (Rampal et al. 2014). A recent report suggested that JAK2 signaling might occur in ET megakaryocytes with CALR mutations; however, the downstream signaling mechanisms other than those involving STAT3 and STAT5 are likely involved in the pathogenesis of MPN with CALR mutations (Lau et al. 2015). Finally, STAT1, STAT3, and STAT5 were not phosphorylated in the MARIMO cell line derived from a patient with ET harboring a CALR mutation (Kollmann et al. 2015). Taken together, these findings suggest the possibility that a lack of STAT3 phosphorylation may be related to the lower NAP score observed in MPN patients with CALR mutations. In patients with JAK2-V617F, NAP expression increases in parallel to the $\mathrm{V} 617 \mathrm{~F}$ allele burden (Basquiera et al. 2007; Vannucchi et al. 2007); however, the relationship between the $C A L R$-mutated allele burden and NAP expression is unknown.

The detailed mechanism that causes CALR mutations and their relationship with a low NAP score remains to be elucidated. Our results reinforce the biological and diagnostic importance of the relationship between the NAP score and MPN. 


\section{Conclusion}

$C A L R$ mutations are one of the major driver mutations in ET and PMF. In addition, the NAP score reflects the pathophysiological status of patients with MPN. We found that the NAP score of patients with CALR mutations ranged from normal to low levels, and that the median NAP score of patients with $C A L R$ mutations was significantly lower than the normal controls. This is the first report of the relationship between the NAP score and CALR mutations, but the detailed mechanism is still unclear. A low NAP score is a unique aspect of MPN patients with $C A L R$ mutations.

\section{Authors' contributions}

TK and NT performed the molecular genetic analysis of all the patient samples; TK performed the statistical analysis; TK, TT, FS, HT, SS, T, YM, HN, HW, and TS provided the patient materials and clinical data; KT and TS contributed advice; TK and TT wrote the manuscript; All authors read and approved the final manuscript.

\section{Author details}

1 Department of Hematology, Kawasaki Medical School, 577 Matsushima, Kurashiki City, Okayama Prefecture 701-0192, Japan. ${ }^{2}$ Department of Laboratory Medicine, Kawasaki Medical School, 577 Matsushima, Kurashiki City, Okayama Prefecture 701-0192, Japan. ${ }^{3}$ Department of Transfusion Medicine and Cell Therapy, Saitama Medical Center, Saitama Medical University, 1981 Kamoda, Kawagoe City, Saitama Prefecture 350-8550, Japan.

\section{Acknowledgements}

The authors would like to thank the patients who agreed to participate in this study. The authors would also like to thank their coworkers for their technical assistance as well as the physicians who provided the patient materials.

\section{Competing interests}

The authors declare that they have no competing interests.

\section{Grant sources}

This work was supported, in part, by a Grant-in-Aid for Scientific Research from the Japan Society for the Promotion of Science and a Kawasaki Medical School Project Grant.

Received: 1 May 2016 Accepted: 14 July 2016

Published online: 22 July 2016

\section{References}

Basquiera AL, Fassetta F, Soria N, Barral JM, Ricchi B, García JJ (2007) Accuracy of leukocyte alkaline phosphatase score to predict JAK2 V617F mutation. Haematologica 92(5):704-705

Baxter E, Scott L, Campbell P, East C, Fourouclas N, Swanton S, Vassiliou G, Bench A, Boyd E, Curtin N (2005) Acquired mutation of the tyrosine kinase JAK2 in human myeloproliferative disorders. Lancet 365(9464):1054-1061

Bendix-Hansen K, Bergmann OJ (1985) Evaluation of neutrophil alkaline phosphatase (NAP) activity in untreated myeloproliferative syndromes and in leukaemoid reactions. Scand J Haematol 35(2):219-224

Campbell PJ, Green AR (2006) The myeloproliferative disorders. N Engl J Med 355(23):2452-2466

James C, Ugo V, Le Couédic J, Staerk J, Delhommeau F, Lacout C, Garçon L, Raslova H, Berger R, Bennaceur-Griscelli A, Villeval JL, Constantinescu SN, Casadevall N, Vainchenker W (2005) A unique clonal JAK2 mutation leading to constitutive signalling causes polycythaemia vera. Nature 434(7037):1144-1148

Kaplow LS (1971) Leukocyte alkaline phosphatase in disease. Crit Rev Clin Lab Sci 2(2):243-278

Klampfl T, Gisslinger H, Harutyunyan AS, Nivarthi H, Rumi E, Milosevic JD, Them NCC, Berg T, Gisslinger B, Pietra D, Chen D, Vladimer Gl, Bagienski
K, Milanesi C, Casetti IC, Sant'Antonio E, Ferretti V, Elena C, Schischlik F, Cleary C (2013) Somatic mutations of calreticulin in myeloproliferative neoplasms. N Engl J Med 369(25):2379-2390

Kollmann K, Nangalia J, Warsch W, Quentmeier H, Bench A, Boyd E, Scott M, Drexler HG, Green AR (2015) MARIMO cells harbor a CALR mutation but are not dependent on JAK2/STAT5 signaling. Leukemia 29(2):494-497

Kondo T, Okuno N, Naruse H, Kishimoto M, Tasaka T, Tsujioka T, Matsuoka A Sugihara T, Tohyama Y, Tohyama K (2008) Valiation of the revised 2008 WHO diagnostic criteria in 75 suspected cases of myeloproliferative neoplasm. Leuk Lymphoma 49(9):1784-1791

Kralovics R, Passamonti F, Buser AS, Teo S, Tiedt R, Passweg JR, Tichelli A, Cazzola M, Skoda RC (2005) A gain-of-function mutation of JAK2 in myeloproliferative disorders. N Engl J Med 352(17):1779-1790

Lau WWY, Hannah R, Green AR, Göttgens B (2015) The JAK-STAT signaling pathway is differentially activated in CALR-positive compared with JAK2V617F-positive ET patients. Blood 125(10):1679-1681

Levine RL, Wadleigh M, Cools J, Ebert BL, Wernig G, Huntly BJP, Boggon TJ, Wlodarska I, Clark JJ, Moore S, Adelsperger J, Koo S, Lee JC, Gabriel S, Mercher T, D'Andrea A, Fröhling S, Döhner K, Marynen P, Vandenberghe $P(2005)$ Activating mutation in the tyrosine kinase JAK2 in polycythemia vera, essential thrombocythemia, and myeloid metaplasia with myelofibrosis. Cancer Cell 7(4):387-397

Nangalia J, Massie CE, Baxter EJ, Nice FL, Gundem G, Wedge DC, Avezov E, Li J, Kollmann K, Kent DG, Aziz A, Godfrey AL, Hinton J, Martincorena I, Van Loo P, Jones AV, Guglielmelli P, Tarpey P, Harding HP, Fitzpatrick JD (2013) Somatic CALR mutations in myeloproliferative neoplasms with nonmutated JAK2. N Engl J Med 369(25):2391-2405

Oku S, Takenaka K, Kuriyama T, Shide K, Kumano T, Kikushige Y, Urata S, Yamauchi T, Iwamoto C, Shimoda HK, Miyamoto T, Nagafuji K, Kishimoto J, Shimoda K, Akashi K (2010) JAK2 V617F uses distinct signalling pathways to induce cell proliferation and neutrophil activation. Br J Haematol 150(3):334-344

Pikman Y, Lee BH, Mercher T, McDowell E, Ebert BL, Gozo M, Cuker A, Wernig G, Moore S, Galinsky I, DeAngelo DJ, Clark JJ, Lee SJ, Golub TR, Wadleigh M, Gilliland DG, Levine RL (2006) MPLW515L is a novel somatic activating mutation in myelofibrosis with myeloid metaplasia. PLOS Med 3(7):e270

Rampal R, Al-Shahrour F, Abdel-Wahab O, Patel JP, Brunel J, Mermel CH, Bass AJ, Pretz J, Ahn J, HricikT, Kilpivaara O, Wadleigh M, Busque L, Gilliland DG, Golub TR, Ebert BL, Levine RL (2014) Integrated genomic analysis illustrates the central role of JAK-STAT pathway activation in myeloproliferative neoplasm pathogenesis. Blood 123(22):e123-e133

Rumi E, Pietra D, Guglielmelli P, Bordoni R, Casetti I, Milanesi C, Sant'Antonio E, Ferretti V, Pancrazzi A, Rotunno G, Severgnini M, Pietrelli A, Astori C, Fugazza E, Pascutto C, Boveri E, Passamonti F, De Bellis G, Vannucchi A, Cazzola M (2013) Acquired copy-neutral loss of heterozygosity of chromosome $1 \mathrm{p}$ as a molecular event associated with marrow fibrosis in MPL-mutated myeloproliferative neoplasms. Blood 121(21):4388-4395

Rumi E, Pietra D, Ferretti V, Klampfl T, Harutyunyan AS, Milosevic JD, Them NCC, Berg T, Elena C, Casetti IC, Milanesi C, Sant'Antonio E, Bellini M, Fugazza E, Renna MC, Boveri E, Astori C, Pascutto C, Kralovics R, Cazzola M (2014) JAK2 or CALR mutation status defines subtypes of essential thrombocythemia with substantially different clinical course and outcomes. Blood 123(10):1544-1551

Saitoh H, Shibata A (1993) Levels of serum granulocyte colony-stimulating factor in patients with chronic myeloid leukemia. Leuk Lymphoma 11(5-6):443-446

Tsushita K, Hotta T, Ichikawa A, Murate T, Saito H (1993) Induction of neutrophil alkaline phosphatase gene expression by granulocyte colony-stimulating factor inchronic myelogenous leukemia. Int J Hematol 57:45-52

Vannucchi AM, Antonioli E, Guglielmelli P, Longo G, Pancrazzi A, Ponziani V, Bogani C, Ferrini PR, Rambaldi A, Guerini V, Bosi A, Barbui T (2007) Prospective identification of high-risk polycythemia vera patients based on JAK2V617F allele burden. Leukemia 21(9):1952-1959

Watari K, Asano S, Shirafuji N, Kodo H, Ozawa K, Takaku F, Kamachi SI (1989) Serum granulocyte colony-stimulating factor levels in healthy volunteers and patients with various disorders as estimated by enzyme immunoassay. Blood 73:117-122

Yuo A, Kitagawa S, Okabe T, Urabe A, Komatsu Y, Itoh S, Takaku F (1987) Recombinant human granulocyte colony-stimulating factor repairs the abnormalities of neutrophils in patients with myelodysplastic syndromes and chronic myelogenous leukemia. Blood 70:404-411 